\title{
The Fate of Neutron Star Binary Mergers
}

\author{
Anthony L. Piro ${ }^{1}$ (D) , Bruno Giacomazzo ${ }^{2,3}$ (D) , and Rosalba Perna ${ }^{4}$ (i) \\ ${ }^{1}$ The Observatories of the Carnegie Institution for Science, 813 Santa Barbara Street, Pasadena, CA 91101, USA; piro@ carnegiescience.edu \\ ${ }^{2}$ Physics Department, University of Trento, via Sommarive 14, I-38123 Trento, Italy \\ ${ }^{3}$ INFN-TIFPA, Trento Institute for Fundamental Physics and Applications, via Sommarive 14, I-38123 Trento, Italy \\ ${ }^{4}$ Department of Physics and Astronomy, Stony Brook University, Stony Brook, NY 11794, USA \\ Received 2017 April 27; accepted 2017 July 7; published 2017 July 27
}

\begin{abstract}
Following merger, a neutron star (NS) binary can produce roughly one of three different outcomes: (1) a stable NS, (2) a black hole (BH), or (3) a supramassive, rotationally supported NS, which then collapses to a BH following angular momentum losses. Which of these fates occur and in what proportion has important implications for the electromagnetic transient associated with the mergers and the expected gravitational wave (GW) signatures, which in turn depend on the high density equation of state (EOS). Here we combine relativistic calculations of NS masses using realistic EOSs with Monte Carlo population synthesis based on the mass distribution of NS binaries in our Galaxy to predict the distribution of fates expected. For many EOSs, a significant fraction of the remnants are NSs or supramassive NSs. This lends support to scenarios in which a quickly spinning, highly magnetized NS may be powering an electromagnetic transient. This also indicates that it will be important for future GW observatories to focus on high frequencies to study the post-merger GW emission. Even in cases where individual GW events are too low in signal to noise to study the post merger signature in detail, the statistics of how many mergers produce NSs versus BHs can be compared with our work to constrain the EOS. To match short gamma-ray-burst (SGRB) $\mathrm{X}$-ray afterglow statistics, we find that the stiffest EOSs are ruled out. Furthermore, many popular EOSs require a significant fraction of $\sim 60 \%-70 \%$ of SGRBs to be from NS-BH mergers rather than just binary NSs.
\end{abstract}

Key words: gamma-ray burst: general - gravitational waves - stars: magnetars - stars: neutron

\section{Introduction}

The collection of binary neutron stars (NSs) in our Galaxy demonstrate that these binaries are sufficiently compact to merge on astrophysically relevant timescales (Martinez et al. 2015). Their mergers are among the most powerful sources of gravitational waves (GWs) in the universe, which are expected to be detected and studied in the coming years by Advanced LIGO (LIGO Scientific Collaboration et al. 2015), Advanced Virgo (Acernese et al. 2015), and KAGRA (Aso et al. 2013). Binary NS mergers are also some of the best candidates for short gamma-ray bursts (SGRBs; Nakar 2007), which is supported by their presence in old stellar environments, nondetection of an associated SN, and large offsets from their host galaxies (Berger 2014). The two main scenarios for generating the central engine are then the formation of a strongly magnetized torus around a spinning black hole $(\mathrm{BH}$; e.g., Popham et al. 1999; Lee \& Ramirez-Ruiz 2007; Rezzolla et al. 2011; Giacomazzo et al. 2013; Ruiz et al. 2016) or the formation of a long-lived magnetar (e.g., Duncan \& Thompson 1992; Dai et al. 2006; Giacomazzo \& Perna 2013; Rowlinson et al. 2013). In addition, post merger, binary NSs have been suggested for a variety of electromagnetic transients that have not been conclusively confirmed. This includes the outflow of neutron-rich material producing a "kilonova" (Metzger 2017; though see Berger et al. 2013 and Tanvir et al. 2013 for a potential case), the spin down of a remnant magnetar producing high energy and later radio emission (Nakar \& Piran 2011; Piro \& Kulkarni 2013; Zhang 2013; Metzger \& Piro 2014), and fast radio bursts (FRBs) from collapsing supramassive NSs (Falcke \& Rezzolla 2014). In other studies, it has been postulated that post-merger magnetars may be an important source of ultrahigh-energy cosmic rays (UHECRs; Piro \& Kollmeier 2016).
In all of these scenarios, a critical question is what is the resulting remnant left over from the binary NS merger. Roughly speaking, there are three main possibilities (Faber \& Rasio 2012). First, the two NSs may have sufficiently low masses to merge and just produce a stable NS. Second, the two merging NSs may have a combined mass above the maximum possible mass for NSs and collapse directly to form a BH. Finally, there is an in-between case where because of the large amount of angular momentum present in the binary, the merged remnant has sufficient spin to initially prevent collapse (the supramassive NS case). Then, only once the remnant NS loses angular momentum (for example, through GW emission, neutrino driven winds, or magnetic dipole spin down), it finally collapses to a $\mathrm{BH}^{5}$ The proportion with which each of these outcomes occurs depends on the initial mass distribution of NS binaries, for which the Galactic sample is our best estimate (Özel et al. 2012; Kiziltan et al. 2013), and the equation of state (EOS) of matter at ultrahigh densities, which continues to be an outstanding unsolved problem in highenergy astrophysics (Lattimer \& Prakash 2001). Eventually, by comparing the expected distribution of outcomes for various EOSs with observed samples of events that are thought to correspond to different outcomes, strict constraints should be made on the possible EOSs.

In the future, the best way to do this will be by following the GW of binary NSs as they coalesce and merge. The coalescence will provide an estimate of each of the NS masses and the post merger signature will probe the remnant object, either corresponding to $\mathrm{BH}$ ringdown if a $\mathrm{BH}$ was promptly

\footnotetext{
5 There is also the fourth potential case in which a remnant NS may be prevented from collapsing for $\sim 10-100 \mathrm{~ms}$ by thermal or differential rotation effects (the "hypermassive NS" case), but we lump this with the direct collapse to $\mathrm{BH}$ scenario for the purposes of this work.
} 
Table 1

Equation of State and Merger Outcome Summary

\begin{tabular}{|c|c|c|c|c|c|}
\hline Equation of State & $\mathrm{H} 4$ & APR4 & GM1 & MS1 & SHT \\
\hline Maximum $M_{g}$ (non-rotating) & 2.01 & 2.16 & 2.39 & 2.75 & 2.77 \\
\hline Maximum $M_{b}$ (non-rotating) & 2.30 & 2.61 & 2.83 & 3.30 & 3.33 \\
\hline Maximum $M_{g}$ (mass-shedding limit) & 2.38 & 2.58 & 2.87 & 3.29 & 3.33 \\
\hline Maximum $M_{b}$ (mass-shedding limit) & 2.70 & 3.07 & 3.36 & 3.89 & 3.94 \\
\hline Spin period (ms) & 0.74 & 0.53 & 0.68 & 0.70 & 0.72 \\
\hline$\beta=T /|W|$ & 0.108 & 0.137 & 0.130 & 0.134 & 0.137 \\
\hline Angular momentum $\left(G M_{\odot}^{2} c^{-1}\right)$ & 3.60 & 4.70 & 5.68 & 7.60 & 7.84 \\
\hline NS remnants (\%) & 0.1 & 6.4 & 44.2 & 99.5 & 99.6 \\
\hline Supramassive NS remnants (\%) & 23.2 & 74.3 & 55.5 & 0.5 & 0.4 \\
\hline BH remnants $(\%)$ & 76.7 & 19.3 & 0.3 & 0.0 & 0.0 \\
\hline
\end{tabular}

Note. All masses are in units of $M_{\odot}$, and the spin periods, $\beta$, and angular momentum are all at the maximum mass, mass-shedding limit.

made or the vibrations of a remnant NS if the EOS will allow this (Bauswein et al. 2012). On the other hand, given the high frequency of the post merger signal, it may be difficult with the advanced era of $\mathrm{GW}$ detectors to study this in full detail. Nevertheless, these experiments should still be able to tell whether a BH or NS remnant formed, which holds important information.

In addition, there may currently be evidence of what the merger outcome is from the early X-ray afterglows of SGRBs. Many of these events ( $~ 50 \%$; Rowlinson et al. 2010) show an "X-ray plateau" that appears to come from extended engine activity, which is then followed by a rapid drop that has been argued to be too steep to be explained by an external shock model. A popular hypothesis for what causes this is that a supramassive NS with a magnetar-like field has been created, which then powers the X-ray plateau until angular momentum loses cause it to collapse to a BH (Fan \& Xu 2006; Gao \& Fan 2006; Rowlinson et al. 2013). If this is the case, then the proportion of SGRBs with X-ray plateaus and the associated timescales should be important constraints for EOSs (Lasky et al. 2014; Lü \& Zhang 2014; Ravi \& Lasky 2014; Gao et al. 2016; Li et al. 2016a).

Motivated by these issues, here we theoretically explore the distribution of NS binary remnant scenarios for a variety of EOSs. In Section 2, we present relativistic calculations of NS masses for realistic EOSs and also discuss the importance of the difference between gravitational and baryonic mass when deriving the final remnant masses. In Section 3, we describe our treatment of the NS mergers, including how we incorporate spin and assess which merger remnants result in supramassive NSs. In Section 4, we use Monte Carlo techniques to merge a population of binary NSs and summarize the proportion of different outcomes. In Section 5, we compare our results to constraints from SGRBs and discuss the various implications of our work, including the impact on GW searches and electromagnetic counterparts. Finally, we conclude in Section 6 with a summary of our results.

\section{NS Models}

We model the NS structures using the EOSs summarized in Table 1. These have been chosen to span a representative range of maximum NS masses, but all with maximum gravitational masses $\gtrsim 2 M_{\odot}$ given current observational limits (Demorest et al. 2010; Antoniadis et al. 2013). For each of these EOSs, we solve for the hydrostatic structure including general relativity and compute a grid of NS models spanning a wide range of masses. We calculate the structures for both a non-rotating NS and a maximally rotating NS for use in treating the merger remnant (further discussed in Section 3). For the latter case, we assume solid-body rotation because magnetic braking eliminates differential rotation on an Alfvén timescale of 10-100 ms (Baumgarte et al. 2000; Shapiro 2000). All models have been computed using the LORENE library and in particular its publicly available codes rotseq and nrotstar. The former can easily compute sequences of non-rotating NSs, while the latter has been used to compute equilibrium configurations at the mass-shedding limit (Stergioulas 2003). The different EOSs were imported in LORENE in a table format either produced by using a piecewise polytropic approximation (APR4, Endrizzi et al. 2016; H4, Kawamura et al. 2016; MS1, Ciolfi et al. 2017), provided by the EOS authors (SHT; Kastaun et al. 2016), or given by the Compose project $\left(\mathrm{GM} 1^{6}\right.$; Glendenning \& Moszkowski 1991; Douchin \& Haensel 2001). This large grid of models gives us, for each mass and EOS, a relation between the gravitational mass

$$
M_{g}=4 \pi \int_{0}^{R} e r^{2} d r
$$

where $e$ is the energy density and $R$ is the NS radius, and the baryonic mass (Shapiro \& Teukolsky 1983)

$$
M_{b}=4 \pi \int_{0}^{R}\left[1-\frac{2 G m(r)}{r c^{2}}\right]^{-1 / 2} \rho r^{2} d r,
$$

where $m(r)$ is the enclosed mass at a given radius and $\rho$ is the rest-mass density (both equations refer to non-rotating models, but similar ones can be derived for rotating models). The important point is that in general $M_{b} \gtrsim M_{g}$ because of the gravitational redshift factor. In the fast rotating limit, we find that $M_{g}$ is greater by $\approx 1.18-1.20$, consistent with the calculations by Breu \& Rezzolla (2016).

The main results of these NS model calculations are summarized in Figure 1. We plot the fractional difference between $M_{b}$ and $M_{g}$ for all of the models we have computed including both non-rotating and maximally rotating structures. This demonstrates that $M_{b}$ can be greater than $M_{g}$ by $\sim 5 \%-20 \%$ with a larger difference for more massive NSs. In addition, not all EOSs have the same difference for a given $M_{g}$. This is because some EOSs have smaller radii and thus are

\footnotetext{
6 http://compose.obspm.fr/
} 


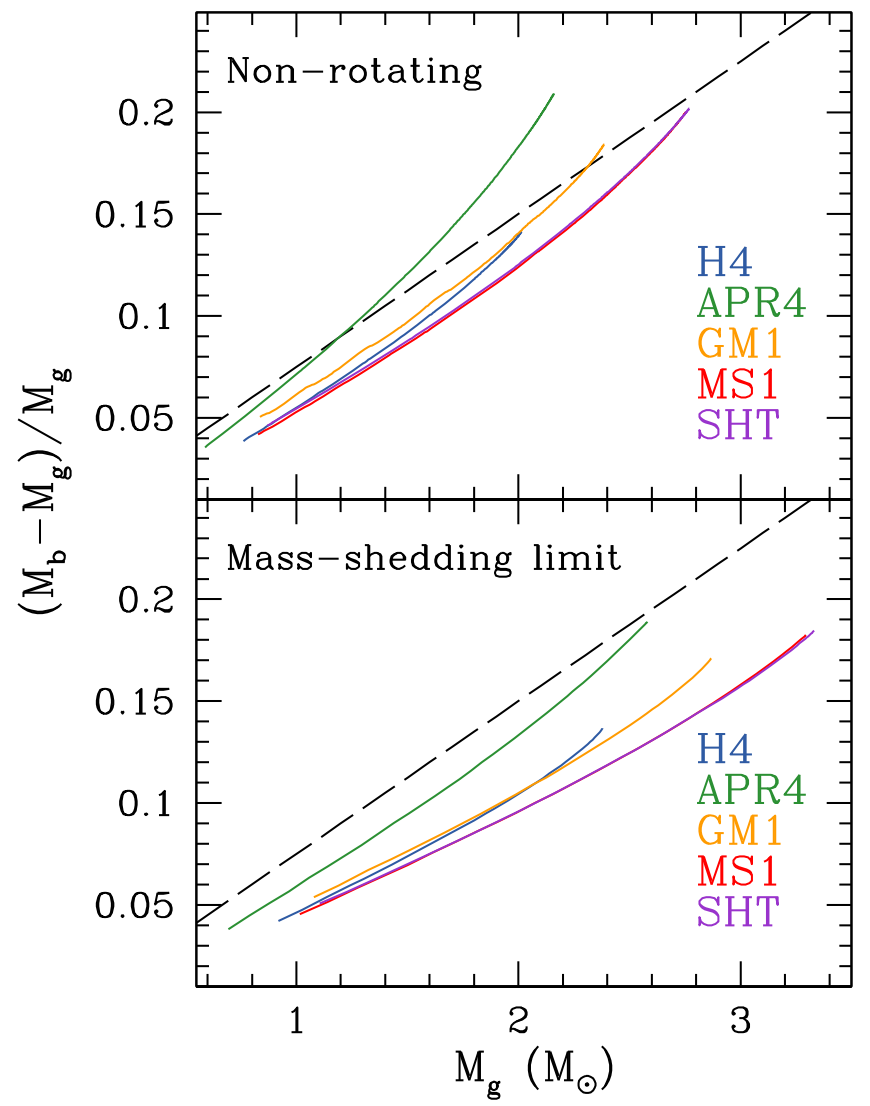

Figure 1. Fractional difference between the baryonic mass $M_{b}$ and the gravitational mass $M_{g}$ as a function of $M_{g}$ for a range of EOSs. We compare both non-rotating (upper panel) and maximally rotating (lower panel) configurations, demonstrating that $M_{b}$ can be $\sim 5 \%-20 \%$ higher than $M_{g}$ The black, dashed line shows Equation (3), an approximation commonly used in the literature (Timmes et al. 1996).

more relativistic (like APR4) than EOSs that give larger radii (like MS1). In addition, the difference is larger for non-rotating versus the rotating models, which will be crucial for understanding the final gravitational mass of merger remnants once it has spun down.

A commonly used approximation for the relation between $M_{g}$ and $M_{b}$ is (Timmes et al. 1996)

$$
M_{b}=M_{g}+0.075 M_{g}^{2},
$$

where each of the masses are in solar units. This is also plotted in Figure 1 in comparison with our more detailed calculations. This demonstrates that the approximation has the correct trend, but generally overpredicts baryonic mass, especially at the mass-shedding limit. It also does not reflect the variation in this relation for different EOSs and at high spin.

\section{Treatment of NS Mergers}

Using this large grid of NS models, we next explore the outcome when they merge. Consider NSs 1 and 2 with gravitational masses $M_{g, 1}$ and $M_{g, 2}$, respectively. During the inspiral, the NSs are not strongly affected by tidal coupling (Bildsten \& Cutler 1992; Kochanek 1992), and thus are not spun up appreciably. For this reason, their structures during this phase are well approximated by our non-spinning NS models.

Next, GWs remove angular momentum from the binary until tidal disruption occurs for the lower mass NS. Assuming
$M_{g, 1}>M_{g, 2}$, this occurs at a semimajor axis (Kopal 1959; adding the $10 \%$ strong gravity correction from Fishbone 1973)

$$
a_{t} \approx 2.4 R_{2}(1 / q+1)^{1 / 3},
$$

where $R_{2}$ is the radius of the less massive NS and $q=M_{g, 2} / M_{g, 1}$. The orbital angular momentum of the binary at this moment is

$$
\begin{aligned}
J_{t} & =\left[G\left(M_{g, 1}+M_{g, 2}\right) a_{t}\right]^{1 / 2} M_{g, 2} \\
& \approx 6.8(1 / q+1)^{1 / 3}\left(\frac{M_{g, 2}}{1.4 M_{\odot}}\right)^{3 / 2}\left(\frac{R_{2}}{10 \mathrm{~km}}\right)^{1 / 2} G M_{\odot}^{2} c^{-1} .
\end{aligned}
$$

This amount of angular momentum must then be incorporated into the merger remnant unless significant high angular momentum material is flung away during the merger. In comparison, we summarize the angular momentum of our maximally spinning models in Table 1 . Accounting for the fact that $(1 / q+1)^{1 / 3} \gtrsim 1.25$ in Equation (5), we see that the amount of angular momentum in the binary at the moment of tidal disruption always exceeds our maximally rotating models. Thus it is reasonable to assume that the merger remnant is near the mass-shedding limit.

Given these estimates, our basic strategy is as follows. If we wish to merge NSs 1 and 2 with gravitational masses $M_{g, 1}$ and $M_{g, 2}$, respectively, we first convert these to their respective baryonic masses $M_{b, 1}$ and $M_{b, 2}$ using our grid of non-rotating models. The total baryonic mass after the merger is then

$$
M_{b, \text { tot }}=M_{b, 1}+M_{b, 2}-M_{\text {lost }}
$$

where $M_{\text {lost }}$ is the mass lost from the system during the merger. In general, this can be due to tidally stripped material, neutrino driven winds from an accretion disk, and neutrinos emitted by the hot remnant, but since the amount of mass lost is expected to be small (Endrizzi et al. 2016), we use a fiducial value of $M_{\text {lost }}=0.01 M_{\odot}$ for this work. Then, using our maximally rotating models, we convert $M_{b \text {,tot }}$ to the total resulting gravitational mass $M_{g \text {,tot }}$. The motivation for using the rotating models when doing this conversion is the large angular momentum in the binary, as discussed above.

In cases where $M_{b, \text { tot }}$ is greater than the maximum $M_{b}$ at the mass-shedding limit for a given EOS, we assume that remnant collapses form a BH. If instead $M_{b \text {,tot }}$ is less than this maximum, but still above the maximum non-rotating $M_{b}$, we assume that the remnant forms a supramassive NS. This NS will only last until it loses sufficient angular momentum to collapse into a $\mathrm{BH}$, potentially via magnetic dipole spin down or GW emission. Finally, if $M_{b, \text { tot }}$ is less than the maximum non-rotating $M_{b}$, then a stable NS is expected to be the final product. In these cases with a stable NS, we perform one additional step of converting the $M_{g}$ in the mass-shedding limit to the (lower) non-spinning $M_{g}$, since this will be the final mass of the remnant once spin down has occurred.

The most likely angular momentum loss mechanisms are magnetic dipole spin down and GW emission. Thus the details of the spin down depend on exactly what magnetic fields are generated and what ellipticity is present, the latter of which can be caused by deforming the NS with internal toroidal magnetic fields. The associated timescales are discussed in detail in Lasky et al. (2014) and Lasky \& Glampedakis (2016), who found that anywhere from $\ll 1 \mathrm{~s}$ to $\sim 10^{4} \mathrm{~s}$ is possible 
depending on the exact parameters chosen. In some cases, it has even been argued that the spin down will proceed in steps to explain the plateau in emission in SGRBs (which we discuss further below), because GWs initially spin down the star on a relatively short timescale to a period of $\sim 10 \mathrm{~ms}$, which is then followed by magnetic dipole emission over the next $\sim 10^{2}-10^{3}$ s (Fan et al. 2013).

In principle, another way to spin down the supramassive NS remnants could be spin-related instabilities. These are typically controlled by the parameter $\beta=T /|W|$, where $T$ is the rotational energy and $W$ is the gravitational binding energy. For $\beta>0.27$, a dynamical bar-mode instability sets in, which leads to mass shedding and spin down back to a stable state (e.g., Shibata et al. 2000). For $0.27>\beta>0.14$, secular instabilities are possible, which can be triggered by viscosity and the gravitational radiation reaction (Chandrasekhar 1970; Friedman \& Schutz 1978; Lai 2001; Gaertig \& Kokkotas 2011). In comparison, our maximally rotating models in Table 1 always have $\beta<0.14$, so neither of these classes of instabilities are expected. Dynamical shear instabilities may operate for $\beta \gtrsim 0.01$ (Passamonti \& Glampedakis 2012; Passamonti et al. 2013), but this requires differential rotation, which is not present in our models. We conclude that GWs and/or magnetic dipole emission are the main spin down mechanisms.

\section{Population Synthesis Results}

Using the procedure outlined in Section 3, we next merge a population of NS binaries using each of the EOSs to find the distribution of outcomes. In each case, we use Monte Carlo techniques to produce $10^{5}$ binaries where the mass distribution of the NSs is drawn from the galactic populations, which is a Gaussian with a mean mass of $\mu=1.32 M_{\odot}$ and a standard deviation of $\sigma=0.11 M_{\odot}$ (Kiziltan et al. 2013). This is in contrast to other studies that attempt to perform the population synthesis from a more first-principles perspective, including stellar models and binary interactions (e.g., Fryer et al. 2015).

In Figure 2, we summarize the results of these calculations for each of the EOSs. In each panel, we plot the distribution of initial (on the left) and final (on the right) baryonic masses for the NS mergers. Even for the initial distributions of $M_{b}$ there are subtle differences for each EOS because of the difference in the conversion of initial $M_{g}$ values (which are all the same) to $M_{b}$ (as summarized in Figure 1).

The vertical dashed lines in Figure 2 divide each panel into three regions, showing the criteria that we use to assess the merger outcome. These are either stable NSs, supramassive NSs (labeled "sNS"), or BHs. These critical masses are given in Table 1 as the maximum $M_{b}$ (non-rotating) and maximum $M_{b}$ (mass-shedding limit) rows. From the proportion of remnants in each of these three regions, we find the outcome percentages, which are summarized in the bottom three rows of Table 1.

These results demonstrate that even over this limited set of EOSs, the distribution of outcomes can vary strongly. For H4, nearly all the mergers eventually make BHs, though many are first supramassive NSs. At the other extreme, MS1 and SHT make almost all stable NSs. This indicates that measuring these distributions, for example, from SGRB features that are from one outcome or another, strong constraints should be placed on the EOS.

Next, in Figure 3, we summarize the corresponding gravitational masses before (left distributions) and after (right

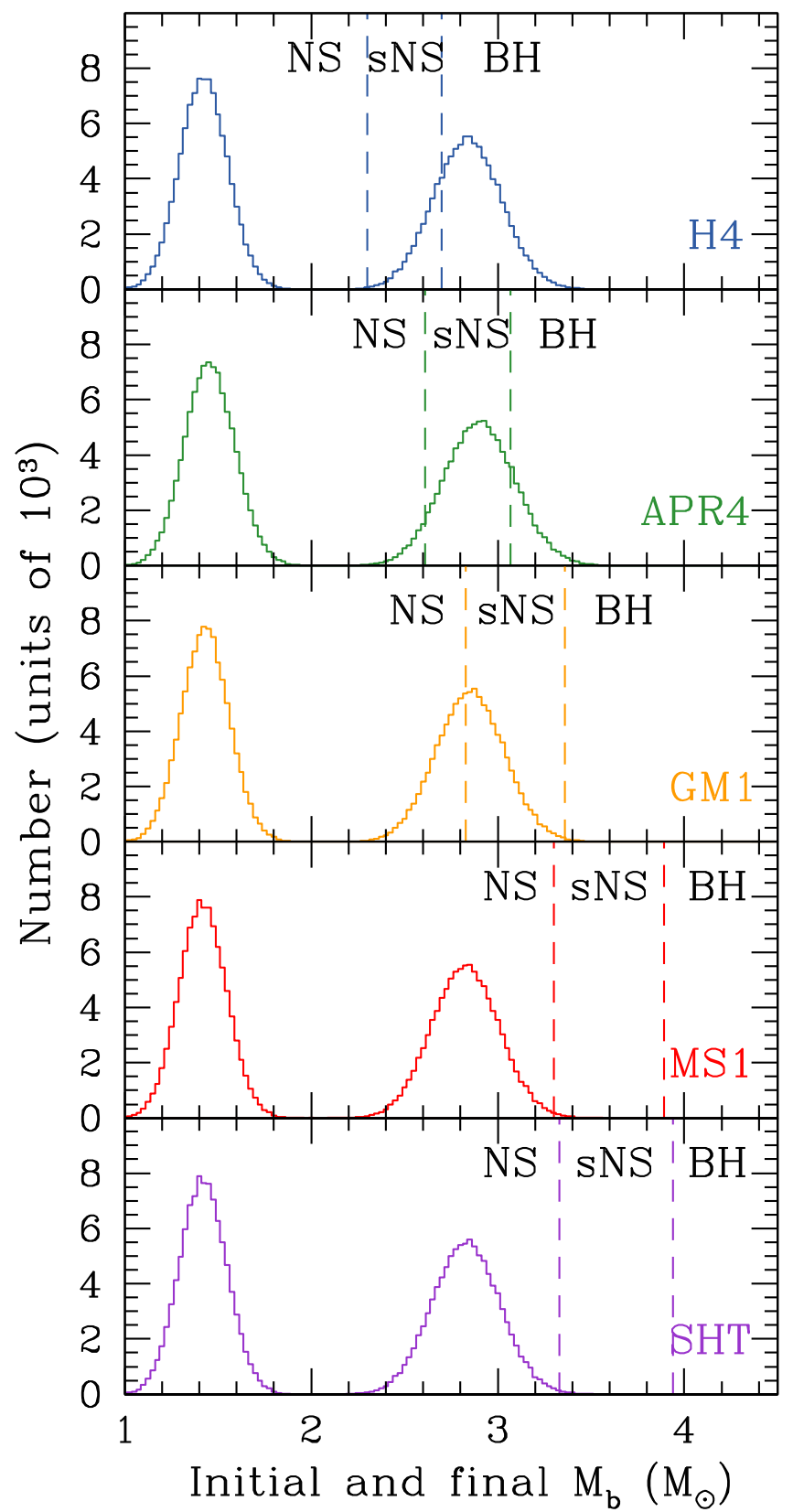

Figure 2. Histograms of the initial (left distribution) and final (right distribution) baryonic masses of NSs after running $10^{5}$ binary models. The different colors correspond to different EOSs as noted in each panel. Vertical dashed lines indicate boundaries between where the final remnant will either be an NS, supramassive NS (labeled as "sNS"), or BH. The percentages of each of these outcomes is summarized in Table 1.

distribution) merger. In this case, we cannot calculate $M_{g}$ in the cases where a $\mathrm{BH}$ is immediately formed, so these instances are removed from the histograms on the right. This is why there is only one dividing line between stable NSs and supramassive NSs. This line is given by taking the maximum, non-rotating $M_{b}$ from Table 1 and converting it to $M_{g}$ in the mass-shedding, which is not given in the table. Eventually, the NSs on the left of the vertical line will spin down to become stable NSs. As this occurs, their $M_{g}$ decreases because they become more relativistic as their radius shrinks. Therefore, the final distribution of $M_{g}$ for the stable NSs are shown with dotted lines. This is further highlighted in Figure 4 to focus on these distributions. 


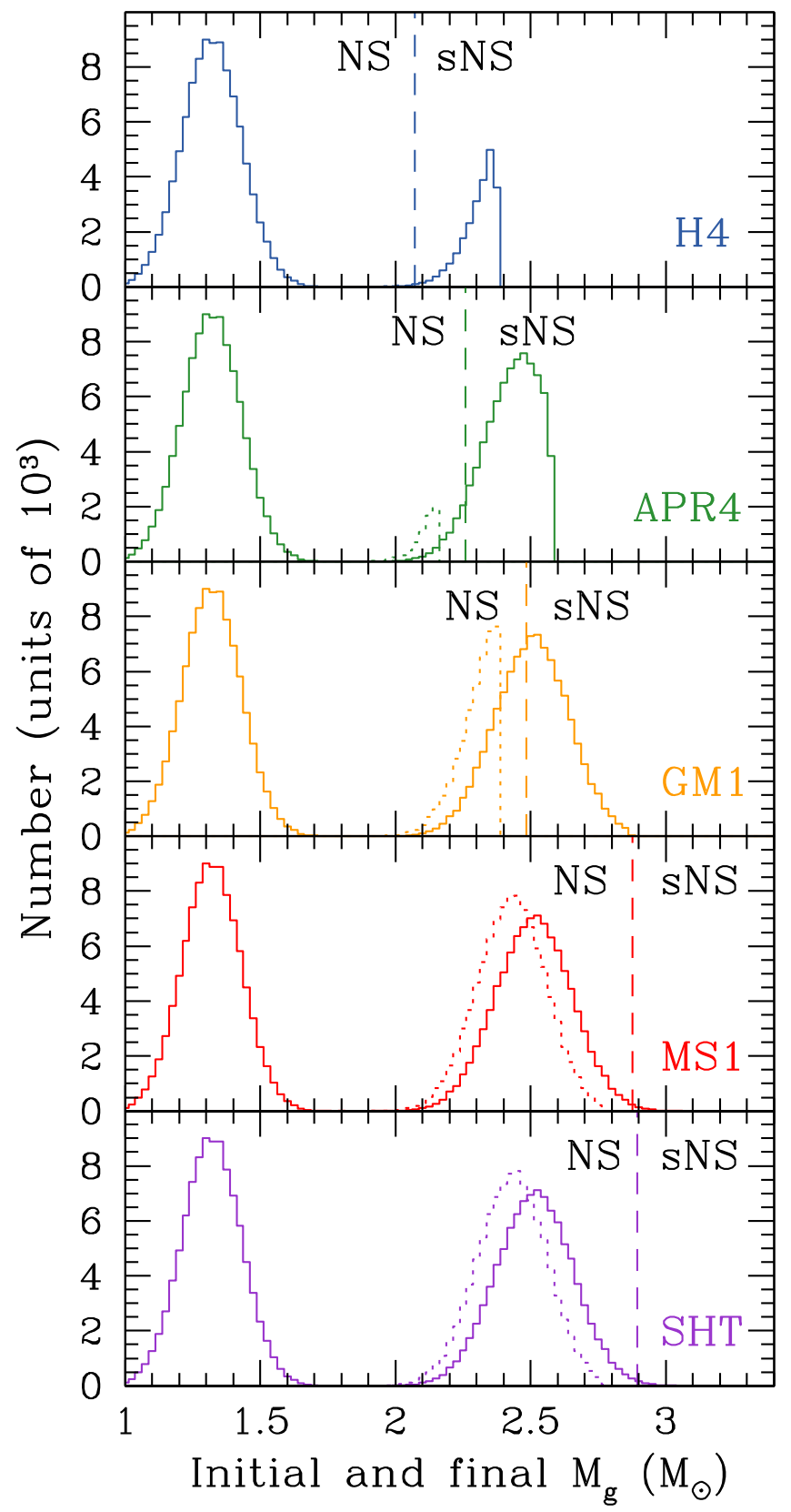

Figure 3. Similar to Figure 2, but now showing the distribution of gravitational masses $M_{g}$. In this case, we cannot convert the remnant $M_{b}$ into $M_{g}$ if the final outcome is a $\mathrm{BH}$, so these are left out of the final distribution. Furthermore, remnants that are stable NSs will have their $M_{g}$ decrease as they spin down. The final, distribution of these, in the limit of zero spin, is shown with the dotted lines.

\section{Comparisons and Implications}

The distribution of fates we find has a number of important connections to SGRBs, other electromagnetic counterparts, GW signatures, and UHECRs. We discuss each of these below with particular emphasis on instances where these observations can be used to constrain the EOS.

\subsection{Short Gamma-ray Bursts}

As introduced in Section 1, several mixed pieces of evidence point to the merger of two compact objects in a binary (NS-NS or NS-BH) as the engine powering SGRBs. In the cases with

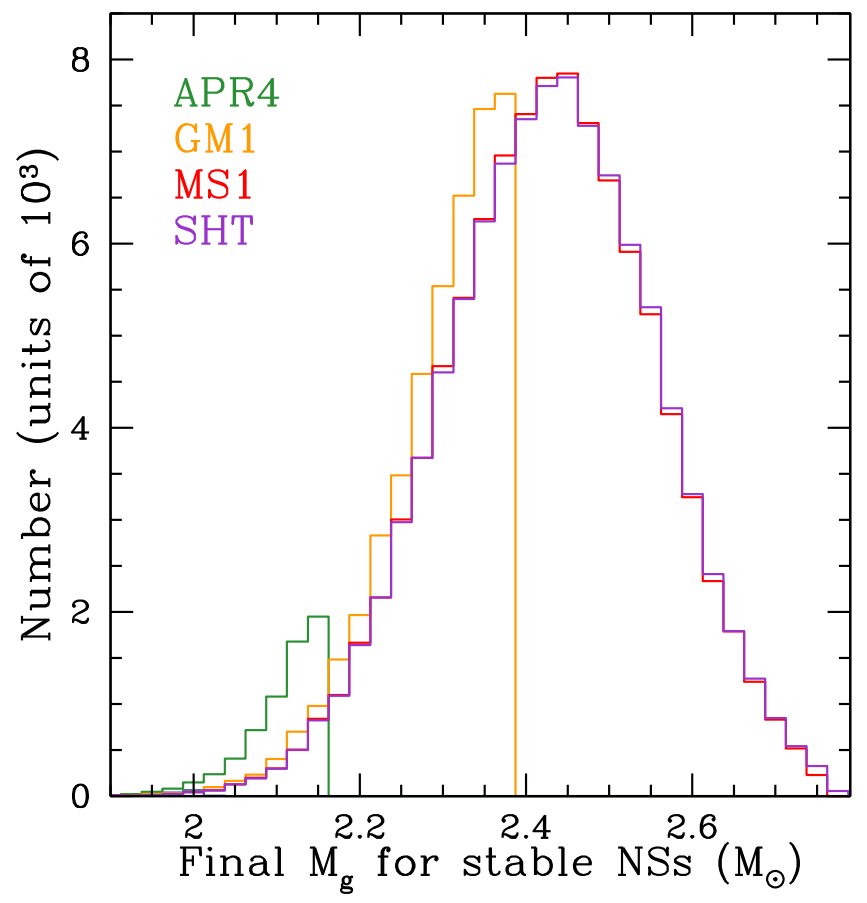

Figure 4. Final $M_{g}$ distribution for the stable NSs following spin down (the same as the dotted lines from Figure 3). H4 is not shown since it does not make appreciable stable NSs.

an NS remnant, it is often thought that there will be a magnetarstrength magnetic field $\left(\sim 10^{14}-10^{15} \mathrm{G}\right)$, either generated by an initially weak field amplified by shear instabilities at the merger interface (Price \& Rosswog 2006; Zrake \& MacFadyen 2013) or by a $\alpha-\Omega$ dynamo in the subsequent neutrino cooling phase (Duncan \& Thompson 1992). This can then potentially power additional emission via magnetic dipole spin down. Indeed, the early observations by the Swift satellite of extended X-ray plateaus followed by either power-law decays or rapid decays suggest that some of these mergers leave behind either a stable NS, or a supramassive NS, which powers this emission until it collapses to a $\mathrm{BH}$ after slowing down.

Using such arguments, Gao et al. (2016) analyzed 96 SGRBs observed with Swift between 2005 January and 2015 October, and found that 21 require a supramassive NS, a fraction of $22 \%$. Our modeling would argue that the EOS H4 matches best with this number. Nevertheless, given the uncertainties in securely identifying whether a supramassive NS is needed and the limited number of events, GM1 might also be possible, and indeed Gao et al. (2016) conclude from their analysis that GM1 is favored. Interestingly, H4 and GM1 give very different predictions for the fraction of stable NSs and prompt BHs.

However, even though we have so far only focused on NSNS binaries, it is possible that a fraction of SGRBs is due to NS-BH mergers as well. Those cases would be added to (and confused with) the sample of prompt collapse and dilute the inferred number of supramassive NSs made from NS-NS binaries. In Figure 5, we plot the fraction of SGRBs that result in NS and supramassive NS remnants as a function of the SGRBs that are due to NS-BH mergers. Quite simply, as the contribution from NS-BH mergers increases, the others must go down. The filled circles denote where the other EOS may match the SGRB constraints. For example, GM1 can get the observed $22 \%$ if $\sim 60 \%$ of SGRBs are from NS-BH mergers, and APR4 could work if $\sim 70 \%$ of SGRBs are from NS-BH 


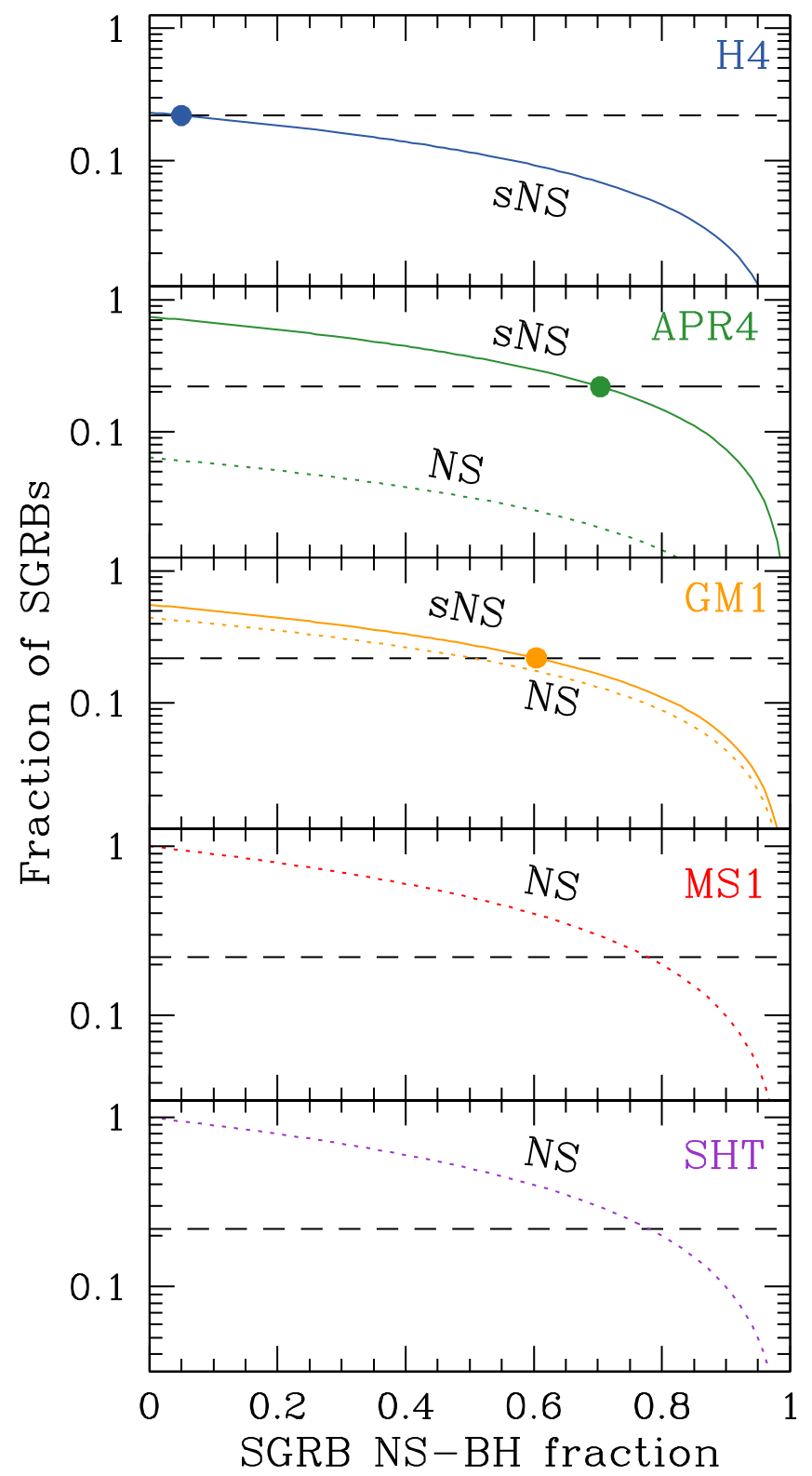

Figure 5. Merger remnant fraction for SGRBs as a function of the fraction of SGRBs that are from NS-BH mergers. For each EOS, the solid lines represent the fraction of supramassive NSs and the dotted lines represent the fraction of stable NSs. The black dashed line at $22 \%$ is the fraction of supramassive NSs as argued by Gao et al. (2016). Filled circles show where we determine each EOS can match this fraction, demonstrating that a significant amount of NS-BH mergers is required in some cases.

mergers. On the other hand, there is no way to produce appreciable supramassive NSs with MS1 or SHT, so the stiffest EOSs appear to be ruled out if the interpretation of these SGRB afterglows is correct.

Although the number of events is lower than in Gao et al. (2016), in the earlier work by Rowlinson et al. (2013), they also argue for a significant fraction of NS remnants from SGRBs. In this case, they conclude that roughly $\sim 64 \%$ of SGRBs form some kind of NS and $\sim 30 \%-60 \%$ of events form a supramassive NS. Similar to what was concluded above, the best fits are then APR4 and GM1, but only if a significant fraction of $\sim 60 \%$ of SGRBs are from NS-BH mergers.

Other groups have argued that the production of a relativistic jet requires SGRB progenitors to collapse promptly to a $\mathrm{BH}$ or at least that the supramassive NS phase must be very short. For example, Murguia-Berthier et al. (2014) argue that collapse must occur within $100 \mathrm{~ms}$ of the initial merger, otherwise baryon contamination prevents the observed high Lorentz factors. Given the large fraction NS or supramassive NS remnants we find, this implies either a fairly soft EOS (with maximum masses in the range of $\approx 2.0-2.2$, Lawrence et al. 2015 , or $\approx 2.3-2.4$, Fryer et al. 2015, depending on the exact distribution of NS masses used) or that a non-negligible fraction of NS-NS mergers do not make SGRBs. The latter possibility is promising for $\mathrm{GW}$ searches, since it implies that there may be many more double NS mergers than even beaming corrected SGRB rates suggest (e.g., Fong et al. 2012). On the other hand, it may mean that there will be many GWs detected without electromagnetic counterparts, which is needed for better localization. That is, unless there are other emission mechanisms beyond just an SGRB, which is what we discuss next.

\subsection{Other Electromagnetic Transients}

Although SGRBs are seen and thought to be associated with NS-NS or NS-BH mergers, there are a number of other electromagnetic transients possibly associated with these events (see the review by Metzger \& Berger 2012). Many of these emission mechanisms are more isotropic than the relativistic jet of an SGRB, and thus are promising for detection, especially in the near future with surveys that have especially rapid cadences, such as the Zwicky Transient Facility (ZTF; Law et al. 2009), the All-sky Automated Survey for Supernovae (Shappee et al. 2014), or the Large Synoptic Survey Telescope (LSST Science Collaboration et al. 2009, depending on the final cadence).

In particular, there are scenarios that require an NS or supramassive NS remnant following the merger. Some examples include the post-merger pulsar wind nebula that can produce an optical and X-ray signature on short timescales of days (Zhang 2013; Metzger \& Piro 2014; Siegel \& Ciolfi 2016a, 2016b; Sun et al. 2017) and radio emission on the timescale of months (Piro \& Kulkarni 2013). Additionally, radio emission may be possible from the small amount of material ejected by the merger $\left(\sim 0.01 M_{\odot}\right)$ that is accelerated by the energy input of a magnetar, which then interacts with the nearby circumstellar medium (Nakar \& Piran 2011). In any case, given that the SGRB comparison favors the EOSs H4, APR4, or GM1, this would argue that these sorts of transients are not uncommon and that future transient surveys can make important constraints on whether these events are occurring. The fraction of SGRBs with radio emission long after the main burst could be combined with our results to constrain the EOS (e.g., Metzger \& Bower 2014; Fong et al. 2016), though one must be careful to include that some SGRBs may be NS-BH mergers (see Figure 5). This could help decide whether H4, APR4, or GM1 is favored since, for the combined fraction of NSs and supramassive NSs, they give very different results of $23.3 \%, 80.7 \%$, and $99.7 \%$, respectively.

Another scenario to consider is whether these mergers make FRBs, a recently discovered class of transients characterized by millisecond bursts of radio emission (Lorimer et al. 2007; Keane et al. 2012; Thornton et al. 2013; Ravi et al. 2015). One idea for generating these is through the collapse of a supramassive NS, which expels its magnetic field as it becomes a BH (Falcke \& Rezzolla 2014). Given our work here, it 
appears that this scenario is quite plausible following a binary NS merger for many EOSs. Nevertheless, it is probably difficult for this to produce the majority of FRBs. Even the highest rate of supramassive NSs by APR4 will only produce roughly $\sim 1 \%$ of FRBs (Rane et al. 2016; Nicholl et al. 2017). Furthermore, the repeating FRB 121102 (Spitler et al. 2014, 2016; Scholz et al. 2016) cannot be reconciled with such a scenario in which the FRB is generated in a single catastrophic event.

\subsection{Impact on $G W$ Searches}

It is expected with the new generation of GW detectors that there will eventually be a detection of binary NS mergers. From these, observations of relatively nearby events could help constrain the EOS via the impact of tidal interactions during the late stages of the merger (e.g., Flanagan \& Hinderer 2008; Read et al. 2009; Baiotti et al. 2010; Hinderer et al. 2010; Bernuzzi et al. 2012). Since, according to our analysis, a significant fraction of NS mergers will produce a long-lived NS remnant, we should expect them to be accompanied by a longduration post-merger GW signal that will be important for EOS constraints as well.

When an NS or supramassive NS is generated, the frequency structure holds detailed clues about the EOS (Dall'Osso et al. 2015; Clark et al. 2016) in the range of $1-4 \mathrm{kHz}$. Due to decreased sensitivity of the advanced GW detectors in this range, detection of these features will either require especially nearby events, potential upgrades to these detectors focused at high frequencies (LIGO Scientific Collaboration et al. 2015; Miller et al. 2015), or the Einstein Telescope (Punturo et al. 2010; Hild et al. 2011). Alternatively, our work shows that the actual fraction of outcomes could also be constraining for the EOS. This would be useful in the case in which there is a large number of events, but their individual post-merger frequency structures have too low signal to noise to constrain the EOS from any given single event. This is likely to be expected because the $\mathrm{BH}$ ringdown for this mass range is much too high for the current generation of GW detectors, while the signature of an NS remnant might still be observed. By simply identifying which events formed NSs (and/or supramassive $\mathrm{NSs}$ ) rather than $\mathrm{BHs}$, the proportion of outcomes could rule out some EOSs using our results as summarized in Table 1.

\subsection{Ultrahigh-energy Cosmic Rays}

Piro \& Kollmeier (2016) argue that rapidly spinning magnetars born from NS mergers are promising sites for the generation of UHECRs (particles with individual energies exceeding $10^{18} \mathrm{eV}$, see reviews by Kotera \& Olinto 2011 and Letessier-Selvon \& Stanev 2011). This is because the shortperiod spin and strong magnetic fields of an NS remnant are able to accelerate particles up to appropriate energies, and the composition of material on and around the NS may naturally explain recent inferences of heavy elements in UHECRs. The low ejecta mass in this scenario (in contrast to UHECRs originating from the centers of supernovae (e.g., Arons 2003; Fang et al. 2012; Kotera et al. 2015) also helps the high-energy neutrino background associated with this scenario to be below current constraints from the IceCube Observatory (The IceCube Collaboration 2011; Aartsen et al. 2013).

A critical uncertainty in this work is whether the fate of the binary NS mergers can explain the detection rate of UHECRs.
For binary NS mergers, Kim et al. (2005) estimates a volumetric rate of $\sim 4 \times 10^{-8}-2 \times 10^{-6} \mathrm{Mpc}^{-3} \mathrm{yr}^{-1}$, which Piro \& Kollmeier (2016) argue could explain the UHECR rate of $\approx 10^{43.5}-10^{44} \mathrm{erg} \mathrm{Mpc}^{-3} \mathrm{yr}^{-1}$ (Waxman 1995; Berezinsky et al. 2006; Katz et al. 2009; Murase \& Takami 2009) if $\sim 10 \%-100 \%$ of the mergers results in NSs or supramassive NSs. In fact, this is accommodated by many of the models we consider, namely H4, APR4, and GM1. Furthermore, the high spin of these remnants is especially important for accelerating particles to the required energies. Paradoxically, the magnetic field need not be at the highest strengths possible. This is because a somewhat lower field of $\sim 10^{13}-10^{14} \mathrm{G}$ (as opposed to $\gtrsim 10^{15} \mathrm{G}$ ) will delay the injection of UHECRs until the surrounding debris has inflated sufficiently and is less likely to impede the particles from leaving the remnant. Ultimately, the detection of binary NS mergers as GW sources will constrain this rate, so that the viability of this model for UHECRs can be better tested.

\section{Discussion and Conclusions}

We have investigated the distribution of outcomes for NS binary mergers by combining relativistic calculations of spinning and non-spinning NS structures for realistic EOSs with the Galactic binary NS mass distribution using Monte Carlo methods. This demonstrated that the fate of NS mergers can vary greatly depending on the EOS, from primarily supramassive NSs and prompt BHs for EOSs like $\mathrm{H} 4$ and primarily stable NSs for EOSs like MS1 and SHT. In still other cases, a fairly even distribution of outcomes is possible.

Perhaps most striking from our results is the non-negligible fraction of NS or supramassive NS remnants expected across all EOSs. At the low end, $\mathrm{H} 4$ predicts $23 \%$ will be supramassive NSs, but when taking NSs and supramassive NSs together, the other four EOSs we consider predict a fraction of $\sim 80 \%-100 \%$. This means that it will be especially important for future $\mathrm{GW}$ observatories to be able to probe the high frequency GW emission in the range of $1-4 \mathrm{kHz}$ to study the frequency structure of the post-merger NS or supramassive NS remnant. Even if particular events have too low in signal to noise to study this frequency spectrum in detail, if the statistics of NS versus $\mathrm{BH}$ remnants can be measured, these proportions can be compared to our results to constrain the EOS.

This means that observations that are sensitive to the ratio of these various outcomes can be useful tools for constraining the EOS. In particular, we have discussed the plateau signature in SGRB X-ray afterglows. If they require $\approx 22 \%$ of events to result in a supramassive NS (as argued by Gao et al. 2016), then H4, APR4, or GM1 can potentially explain this. Interestingly, if one of these latter two EOSs end up being correct, then a significant fraction of $\sim 60 \%-70 \%$ of SGRBs must be from NS-BH mergers. The inference of such a large number of NS-BH mergers is exciting given that there has never been a direct observation of such a system. This has a number of important implications. First, the rate of SGRBs (corrected for beaming, as estimated in Fong et al. 2012) could be greater than the rate of just binary NS mergers (e.g., Kalogera et al. 2004), and we may expect NS-BH mergers to be a significant $\mathrm{GW}$ source in the near future (Maselli \& Ferrari 2014; Li et al. 2016b, 2016c). Next, NS-BH mergers cannot have $\mathrm{BH}$ masses that are too large, otherwise there may not be sufficient material for an accretion disk to power the prompt SGRB emission $\left(\lesssim 8-10 M_{\odot}\right.$, Shibata \& 
Taniguchi 2011; Foucart 2012; Rosswog 2015). This affects the production of $r$-process material from the mergers and the rate of SGRBs needed to produce the observed abundances (for reviews, see Arnould et al. 2007; Qian \& Wasserburg 2007; Sneden et al. 2008; Thielemann et al. 2011). The luminosity of the radioactive electromagnetic transients from compact mergers (e.g., kilonovae; Metzger 2017) would also be affected, which will be studied by the upcoming wide field surveys that are sensitive to short timescale transients (e.g., ZTF; Law et al. 2009),

We thank Paul Lasky, Enrico Ramirez-Ruiz, Luciano Rezzolla, and Bing Zhang for helpful feedback on previous drafts. We thank the OCIW Distinguished Visitor Program at the Carnegie Observatories, which funded R.P.'s visit, where the initial ideas for this work were developed. R.P. acknowledges partial support from the NSF award AST-1616157. B.G. acknowledges partial support from "NewCompStar" COST Action MP1304 and from MIUR FIR grant No. RBFR13QJYF. Some of the computations were performed on the MIES cluster of the Carnegie Observatories, which was made possible by a grant from the Ahmanson Foundation.

\section{ORCID}

Anthony L. Piro (1) https://orcid.org/0000-0001-6806-0673

Bruno Giacomazzo (i) https://orcid.org/0000-0002-6947-4023

Rosalba Perna (i) https://orcid.org/0000-0002-3635-5677

\section{References}

Aartsen, M. G., Abbasi, R., Abdou, Y., et al. 2013, ApJ, 779, 132 Acernese, F., Agathos, M., Agatsuma, K., et al. 2015, CQGra, 32, 024001

Antoniadis, J., Freire, P. C. C., Wex, N., et al. 2013, Sci, 340, 448

Arnould, M., Goriely, S., \& Takahashi, K. 2007, PhR, 450, 97

Arons, J. 2003, ApJ, 589, 871

Aso, Y., Michimura, Y., Somiya, K., et al. 2013, PhRvD, 88, 043007

Baiotti, L., Damour, T., Giacomazzo, B., Nagar, A., \& Rezzolla, L. 2010 PhRvL, 105, 261101

Baumgarte, T. W., Shapiro, S. L., \& Shibata, M. 2000, ApJL, 528, L29

Bauswein, A., Janka, H.-T., Hebeler, K., \& Schwenk, A. 2012, PhRvD, 86, 063001

Berezinsky, V., Gazizov, A., \& Grigorieva, S. 2006, PhRvD, 74, 043005

Berger, E. 2014, ARA\&A, 52, 43

Berger, E., Fong, W., \& Chornock, R. 2013, ApJL, 774, L23

Bernuzzi, S., Nagar, A., Thierfelder, M., \& Brügmann, B. 2012, PhRvD, 86, 044030

Bildsten, L., \& Cutler, C. 1992, ApJ, 400, 175

Breu, C., \& Rezzolla, L. 2016, MNRAS, 459, 646

Chandrasekhar, S. 1970, ApJ, 161, 561

Ciolfi, R., Kastaun, W., Giacomazzo, B., et al. 2017, PhRvD, 95, 063016

Clark, J. A., Bauswein, A., Stergioulas, N., \& Shoemaker, D. 2016, CQGra, 33 , 085003

Dai, Z. G., Wang, X. Y., Wu, X. F., \& Zhang, B. 2006, Sci, 311, 1127

Dall'Osso, S., Giacomazzo, B., Perna, R., \& Stella, L. 2015, ApJ, 798, 25

Demorest, P. B., Pennucci, T., Ransom, S. M., Roberts, M. S. E., \& Hessels, J. W. T. 2010, Natur, 467, 1081

Douchin, F., \& Haensel, P. 2001, A\&A, 380, 151

Duncan, R. C., \& Thompson, C. 1992, ApJL, 392, L9

Endrizzi, A., Ciolfi, R., Giacomazzo, B., Kastaun, W., \& Kawamura, T. 2016, CQGra, 33, 164001

Faber, J. A., \& Rasio, F. A. 2012, LRR, 15, 8

Falcke, H., \& Rezzolla, L. 2014, A\&A, 562, A137

Fan, Y.-Z., Wu, X.-F., \& Wei, D.-M. 2013, PhRvD, 88, 067304

Fan, Y.-Z., \& Xu, D. 2006, MNRAS, 372, L19

Fang, K., Kotera, K., \& Olinto, A. V. 2012, ApJ, 750, 118

Fishbone, L. G. 1973, ApJ, 185, 43

Flanagan, É É, \& Hinderer, T. 2008, PhRvD, 77, 021502

Fong, W., Berger, E., Margutti, R., et al. 2012, ApJ, 756, 189

Fong, W., Metzger, B. D., Berger, E., \& Özel, F. 2016, ApJ, 831, 141
Foucart, F. 2012, PhRvD, 86, 124007

Friedman, J. L., \& Schutz, B. F. 1978, ApJ, 222, 281

Fryer, C. L., Belczynski, K., Ramirez-Ruiz, E., et al. 2015, ApJ, 812, 24

Gaertig, E., \& Kokkotas, K. D. 2011, PhRvD, 83, 064031

Gao, H., Zhang, B., \& Lü, H.-J. 2016, PhRvD, 93, 044065

Gao, W.-H., \& Fan, Y.-Z. 2006, ChJA\&A, 6, 513

Giacomazzo, B., \& Perna, R. 2013, ApJL, 771, L26

Giacomazzo, B., Perna, R., Rezzolla, L., Troja, E., \& Lazzati, D. 2013, ApJL, 762, L18

Glendenning, N. K., \& Moszkowski, S. A. 1991, PhRvL, 67, 2414

Hild, S., Abernathy, M., Acernese, F., et al. 2011, CQGra, 28, 094013

Hinderer, T., Lackey, B. D., Lang, R. N., \& Read, J. S. 2010, PhRvD, 81, 123016

Kalogera, V., Kim, C., Lorimer, D. R., et al. 2004, ApJL, 601, L179

Kastaun, W., Ciolfi, R., \& Giacomazzo, B. 2016, PhRvD, 94, 044060

Katz, B., Budnik, R., \& Waxman, E. 2009, JCAP, 3, 020

Kawamura, T., Giacomazzo, B., Kastaun, W., et al. 2016, PhRvD, 94, 064012

Keane, E. F., Stappers, B. W., Kramer, M., \& Lyne, A. G. 2012, MNRAS, 425, L71

Kim, C., Kalogera, V., Lorimer, D. R., Ihm, M., \& Belczynski, K. 2005, in ASP Conf. Ser. 328, Binary Radio Pulsars, ed. F. A. Rasio \& I. H. Stairs (San Francisco, CA: ASP), 83

Kiziltan, B., Kottas, A., De Yoreo, M., \& Thorsett, S. E. 2013, ApJ, 778, 66

Kochanek, C. S. 1992, ApJ, 398, 234

Kopal, Z. 1959, Close Binary Systems (London: Chapman and Hall)

Kotera, K., Amato, E., \& Blasi, P. 2015, JCAP, 8, 026

Kotera, K., \& Olinto, A. V. 2011, ARA\&A, 49, 119

Lai, D. 2001, in AIP Conf. Ser. 575, Astrophysical Sources for Ground-Based Gravitational Wave Detectors, ed. J. M. Centrella (Melville, NY: AIP), 246

Lasky, P. D., \& Glampedakis, K. 2016, MNRAS, 458, 1660

Lasky, P. D., Haskell, B., Ravi, V., Howell, E. J., \& Coward, D. M. 2014, PhRvD, 89, 047302

Lattimer, J. M., \& Prakash, M. 2001, ApJ, 550, 426

Law, N. M., Kulkarni, S. R., Dekany, R. G., et al. 2009, PASP, 121, 1395

Lawrence, S., Tervala, J. G., Bedaque, P. F., \& Miller, M. C. 2015, ApJ, 808, 186

Lee, W. H., \& Ramirez-Ruiz, E. 2007, NJPh, 9, 17

Letessier-Selvon, A., \& Stanev, T. 2011, RvMP, 83, 907

Li, A., Zhang, B., Zhang, N.-B., et al. 2016a, PhRvD, 94, 083010

Li, X., Hu, Y.-M., Fan, Y.-Z., \& Wei, D.-M. 2016b, ApJ, 827, 75

Li, X., Hu, Y.-M., Jin, Z.-P., Fan, Y.-Z., \& Wei, D.-M. 2016c, arXiv:1611.01760

LIGO Scientific Collaboration, Aasi, J., Abbott, B. P., et al. 2015, CQGra, 32, 074001

Lorimer, D. R., Bailes, M., McLaughlin, M. A., Narkevic, D. J., \& Crawford, F. 2007, Sci, 318, 777

LSST Science Collaboration, Abell, P. A., Allison, J., et al. 2009, arXiv:0912.0201

Lü, H.-J., \& Zhang, B. 2014, ApJ, 785, 74

Martinez, J. G., Stovall, K., Freire, P. C. C., et al. 2015, ApJ, 812, 143

Maselli, A., \& Ferrari, V. 2014, PhRvD, 89, 064056

Metzger, B. D. 2017, LRR, 20, 3

Metzger, B. D., \& Berger, E. 2012, ApJ, 746, 48

Metzger, B. D., \& Bower, G. C. 2014, MNRAS, 437, 1821

Metzger, B. D., \& Piro, A. L. 2014, MNRAS, 439, 3916

Miller, J., Barsotti, L., Vitale, S., et al. 2015, PhRvD, 91, 062005

Murase, K., \& Takami, H. 2009, ApJL, 690, L14

Murguia-Berthier, A., Montes, G., Ramirez-Ruiz, E., De Colle, F., \& Lee, W. H. 2014, ApJL, 788, L8

Nakar, E. 2007, PhR, 442, 166

Nakar, E., \& Piran, T. 2011, Natur, 478, 82

Nicholl, M., Williams, P. K. G., Berger, E., et al. 2017, ApJ, 843, 84

Özel, F., Psaltis, D., Narayan, R., \& Santos Villarreal, A. 2012, ApJ, 757, 55

Passamonti, A., Gaertig, E., Kokkotas, K. D., \& Doneva, D. 2013, PhRvD, 87, 084010

Passamonti, A., \& Glampedakis, K. 2012, MNRAS, 422, 3327

Piro, A. L., \& Kollmeier, J. A. 2016, ApJ, 826, 97

Piro, A. L., \& Kulkarni, S. R. 2013, ApJL, 762, L17

Popham, R., Woosley, S. E., \& Fryer, C. 1999, ApJ, 518, 356

Price, D. J., \& Rosswog, S. 2006, Sci, 312, 719

Punturo, M., Abernathy, M., Acernese, F., et al. 2010, CQGra, 27, 084007

Qian, Y.-Z., \& Wasserburg, G. J. 2007, PhR, 442, 237

Rane, A., Lorimer, D. R., Bates, S. D., et al. 2016, MNRAS, 455, 2207

Ravi, V., \& Lasky, P. D. 2014, MNRAS, 441, 2433

Ravi, V., Shannon, R. M., \& Jameson, A. 2015, ApJL, 799, L5

Read, J. S., Markakis, C., Shibata, M., et al. 2009, PhRvD, 79, 124033

Rezzolla, L., Giacomazzo, B., Baiotti, L., et al. 2011, ApJL, 732, L6

Rosswog, S. 2015, IJMPD, 24, 1530012 
Rowlinson, A., O’Brien, P. T., Metzger, B. D., Tanvir, N. R., \& Levan, A. J. 2013, MNRAS, 430, 1061

Rowlinson, A., O'Brien, P. T., Tanvir, N. R., et al. 2010, MNRAS, 409, 531

Ruiz, M., Lang, R. N., Paschalidis, V., \& Shapiro, S. L. 2016, ApJL, 824, L6

Scholz, P., Spitler, L. G., Hessels, J. W. T., et al. 2016, ApJ, 833, 177

Shapiro, S. L. 2000, ApJ, 544, 397

Shapiro, S. L., \& Teukolsky, S. A. 1983, Black Holes, White Dwarfs, and Neutron Stars: The Physics of Compact Objects (New York: WileyInterscience)

Shappee, B. J., Prieto, J. L., Grupe, D., et al. 2014, ApJ, 788, 48

Shibata, M., Baumgarte, T. W., \& Shapiro, S. L. 2000, ApJ, 542, 453

Shibata, M., \& Taniguchi, K. 2011, LRR, 14, 6

Siegel, D. M., \& Ciolfi, R. 2016a, ApJ, 819, 14
Siegel, D. M., \& Ciolfi, R. 2016b, ApJ, 819, 15

Sneden, C., Cowan, J. J., \& Gallino, R. 2008, ARA\&A, 46, 241

Spitler, L. G., Cordes, J. M., Hessels, J. W. T., et al. 2014, ApJ, 790, 101

Spitler, L. G., Scholz, P., Hessels, J. W. T., et al. 2016, Natur, 531, 202

Stergioulas, N. 2003, LRR, 6, 3

Sun, H., Zhang, B., \& Gao, H. 2017, ApJ, 835, 7

Tanvir, N. R., Levan, A. J., Fruchter, A. S., et al. 2013, Natur, 500, 547

The IceCube Collaboration 2011, arXiv:1111.2736

Thielemann, F.-K., Arcones, A., Käppeli, R., et al. 2011, PrPNP, 66, 346

Thornton, D., Stappers, B., Bailes, M., et al. 2013, Sci, 341, 53

Timmes, F. X., Woosley, S. E., \& Weaver, T. A. 1996, ApJ, 457, 834

Waxman, E. 1995, PhRvL, 75, 386

Zhang, B. 2013, ApJL, 763, L22

Zrake, J., \& MacFadyen, A. I. 2013, ApJL, 769, L29 